\title{
Tendências Genéticas em Características de Desempenho de Suínos das Raças Large White, Landrace e Duroc ${ }^{1}$
}

\author{
André Ribeiro Corrêa da Costa ${ }^{2}$, Paulo Sávio Lopes ${ }^{4,6}$ Robledo de Almeida Torres ${ }^{4,6}$, Ricardo \\ Frederico Euclydes ${ }^{4}$, Adair José Regazzi ${ }^{4,6}$, Martinho de Almeida e Silva ${ }^{5,6}$, Aldrin Vieira Pires ${ }^{3,6}$
}

RESUMO - Dados de suínos das raças Large White (LW), Landrace (LD) e Duroc (DU) foram usados para estimar as tendências genéticas para as características peso ajustado para 70 dias (PA70) em kg, ganho de peso diário (GPD), em g, e espessura de toucinho (ET), em cm. As estimativas de tendências genéticas foram obtidas por meio da regressão das médias dos valores genéticos das características, em função do ano de nascimento do animal. As estimativas de tendência genética para PA70 ( $L W=-0,22 ; \mathrm{LD}=-0,24 ; \mathrm{DU}=-0,57)$ foram negativas, em razão, possivelmente, da maior ênfase dada à seleção de GPD e ET e das correlações entre essas características. As estimativas de tendência genética para ganho de peso diário GPD $(\mathrm{LW}=14,11 ; \mathrm{LD}=9,81 ; \mathrm{DU}=2,75)$ e espessura de toucinho $(\mathrm{LW}=-0,07 ; \mathrm{LD}=-0,05 ; \mathrm{DU}=$ -0,04), estão de acordo com os objetivos do programa e com a correlação existente entre estas características.

Palavras-chave: BLUP, suíno, tendência genética

\section{Genetic Trends in Performance Traits of Large White, Landrace and Duroc Swine Breeds}

\begin{abstract}
Data of Large White (LW), Landrace (LD) and Duroc (DU) swine were used to estimate the genetic trends for adjusted weight at 70-days old (AW70) in kg, average daily gain (ADG) in g and backfat thickness (BT) in $\mathrm{cm}$. The genetic trends were estimated by regression of the averages of trait genetic values in function of the animal birth year. The estimates of the genetic trend for $\mathrm{AW} 70(\mathrm{LW}=-0.22$; $\mathrm{LD}=-0.24 ; \mathrm{DU}=-0.57$ ) were negative, possibly due to a greater emphasis given in the selection process for $\mathrm{ADG}$ and $\mathrm{BT}$ and to the correlations between these traits. The estimates of the genetic trend for ADG $(\mathrm{LW}=14.11 ; \mathrm{LD}=9.81 ; \mathrm{DU}=2.75)$ and $\mathrm{BT}(\mathrm{LW}=-0.07 ; \mathrm{LD}=-0.05 ; \mathrm{DU}$ $=-0.04)$ are in accordance with the objectives of the genetic program and with the existing correlation between these traits as well.
\end{abstract}

Key Words: BLUP, genetic trend, swine

\section{Introdução}

A estimação de tendências genéticas em uma população permite visualizar a eficácia dos procedimentos de seleção. Segundo HUDSON e KENNEDY (1985), permite monitorar a eficácia das estratégias de melhoramento e assegurar que a pressão de seleção seja direcionada para as características de importância econômica, além de auxiliar na definição dos objetivos da seleção.

Em melhoramento animal, em que se trabalha com grande número de animais e é difícil controlar as flutuações ambientais, o maior problema é a estimação de tendências genéticas livres do viés causado pelo ambiente.

HILL (1972a e 1972b) propôs dois procedimentos para se remover a influência ambiental: por meio da utilização de grupos-controle ou pelo esquema de seleção divergente. Porém, quando se desenvolve melhoramento com fins comerciais, esses dois procedimentos são difíceis de serem estabelecidos, principalmente em razão do custo de se manterem as populações-controle.

SORENSEN e KENNEDY (1984), ao usarem dados simulados, demonstraram que, sob determinadas condições, as equações de modelos mistos dispensam o uso de populações-controle como meio para separar, adequadamente, a tendência fenotípica em componentes genéticos e ambientais. Segundo esses autores, se as variâncias das características, antes da seleção, forem conhecidas, a seleção for função linear dos dados e a matriz de parentesco for completa, ou seja, se todos os animais com dados de desempenho tiverem pai e mãe conhecidos, então a tendência genética pode ser estimada por meio da metodologia de modelos mistos.

\footnotetext{
${ }^{1}$ Parte da Tese de mestrado primeiro autor.

2 MS em Zootecnia, UFV.

3 Estudante de Doutorado da UFV. E-mail: avpires@alunos.ufv.br

${ }^{4}$ Professor da UFV. E-mail: plopes@mail.ufv.br, rtorres@mail.ufv.br, rbaja@mail.ufv.br, adairreg@mail.ufv.br

5 Professor da UFMG. E-mail: martinho@vet.ufmg.br

6 Bolsista do CNPq.
} 
BLAIR e POLLAK (1984), ao trabalharem com uma linha-controle e uma linha selecionada, utilizaram três metodologias para estimar a superioridade genética da linha selecionada, a saber: 1) O desvio do fenótipo anual predito da linha selecionada, em relação ao fenótipo anual predito da linha-controle; 2) $\mathrm{O}$ desvio do fenótipo anual predito da linha selecionada em relação à estimativa do ano da linha-controle; e 3 ) A média do valor genético anual da linha selecionada para estimar a superioridade genética da linha selecionada. Observaram que a média do valor genético anual da linha selecionada dependia da herdabilidade e promovia melhor separação da tendência fenotípica em genética e ambiental, concluindo que esse tipo de análise pode ser usado na estimação de tendências genéticas, principalmente em dados provenientes de rebanhos de melhoramento para fins comerciais, nos quais normalmente não se estabelecem linhas-controle.

O objetivo do presente trabalho foi estimar tendências genéticas para o peso ajustado para 70 dias, ganho de peso diário e espessura de toucinho de suínos Large White, Landrace e Duroc.

\section{Material e Métodos}

Os dados utilizados são provenientes de animais da empresa COOPERCENTRAL, situada no município de Chapecó-SC, das raças Landrace, Large White e Duroc. O banco de dados foi constituído por registros de 6237 animais, para peso ajustado aos 70 dias (PA70), e de 3.184 animais, para ganho de peso diário dos 70 aos 140 dias de idade (GPD)e espessura de toucinho aos 140 dias de idade (ET), para a raça Landrace; de 8432 animais, para PA70, e 4965 animais, para GPD e ET, na raça Large White; e de 4696 animais, para PA70, e 1823 animais, para GPD e ET, na raça Duroc. As médias aritméticas e os desvios-padrão das características são apresentados na Tabela 1. As informações referem-se ao período de 1995 a 1997.

O arquivo de pedigree possuía 8651 animais da raça Landrace, 11.184 animais da Large White e 6835 animais da Duroc. Esse arquivo constava da identificação do indivíduo, de seu pai e de sua mãe.

Os animais foram reunidos em grupos contemporâneos formados pela combinação da estação do ano (1 - dezembro a fevereiro; 2 - março a maio; 3 - junho a agosto; e 4 - setembro a novembro) com o ano de nascimento e o sexo, para a raça Duroc, e da combinação destes com a granja, para as raças Landrace e Large White. O tamanho da leitegada, no desmame; a idade, ao final da fase de creche; e o peso, ao final do teste foram usados, respectivamente, como covariáveis para peso ajustado aos 70 dias, ganho de peso diário e espessura de toucinho.

Utilizou-se o seguinte modelo estatístico, em análise conjunta das características:

$$
\mathrm{y}=\mathrm{X} \beta+\mathrm{Z} \mu+\mathrm{Wp}+\varepsilon
$$

em que y é vetor dos dados para as três características; $X$, matriz de incidência dos efeitos fixos e covariáveis; $\beta$, vetor dos efeitos fixos de grupo contemporâneo e covariáveis, para as três características; Z, matriz de incidência dos valores genéticos; $\mu$, vetor dos valores genéticos dos indivíduos para as três características; $\mathrm{W}$, matriz de incidência dos efeitos de leitegada; $p$, vetor dos efeitos aleatórios de leitegada, para a três características; $\varepsilon$, vetor dos erros aleatórios.

Admitiu-se que $y, \mu, p$ e $\varepsilon$ tinham distribuição normal multivariada,

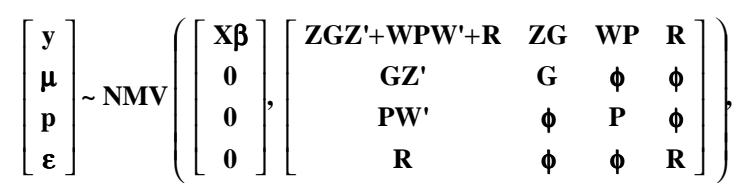

em que $G$ é $A \otimes G_{0}$, sendo $A$, matriz do numerador dos coeficientes de parentesco de Wright; $\mathrm{G}_{0}$, matriz de (co)variâncias genéticas aditivas; $\mathrm{e} \otimes$, operador do produto direto; $\mathrm{P}=\mathrm{I} \otimes \mathrm{P}_{0}$, sendo I, matriz identidade; e $\mathrm{P}_{0}$, matriz de (co)variâncias do efeito de leitegada; $\mathrm{R}=\mathrm{I} \otimes \mathrm{R}_{0}$, sendo $\mathrm{I}$, matriz identidade; e $\mathrm{R}_{0}$, matriz de (co)variâncias residuais; $\phi=$ matriz de zeros.

Para obtenção das predições dos valores genéticos, utilizou-se o programa MTDFREML (BOLDMAN et al., 1995).

As estimativas de tendências genéticas foram obtidas por meio da regressão das médias dos valores genéticos das características, em função do ano de nascimento dos animais.

Tabela 1 - Médias aritméticas e desvios-padrão das características por raça

Table 1 - Arithmetic means and standard deviations by trait and breed

\begin{tabular}{lccc}
\hline Raça & PA70 $(\mathrm{kg})$ & GPD $(\mathrm{g})$ & ET $(\mathrm{cm})$ \\
Breed & $A W 70(\mathrm{~kg})$ & $A D G(\mathrm{~g})$ & $B T(\mathrm{~cm})$ \\
\hline Large White & $25,31 \pm 5,27$ & $858,83 \pm 110,13$ & $1,65 \pm 0,47$ \\
Landrace & $25,79 \pm 5,26$ & $824,43 \pm 112,23$ & $1,71 \pm 0,58$ \\
Duroc & $18,98 \pm 4,96$ & $800,88 \pm 89,65$ & $1,39 \pm 0,28$ \\
\hline
\end{tabular}

PA70 = Peso ajustado aos 70 dias (AW70 $=$ Adjusted weight at 70 -days old $)$. $\mathrm{GPD}=$ Ganho de peso diário $(A D G=$ Average daily gain $)$.

$\mathrm{ET}=$ Espessura de toucinho $(B T=$ Backfat thickness $)$. 


\section{Resultados e Discussão}

As estimativas das tendências genéticas anuais do peso aos 70 dias $(\mathrm{kg})$, do ganho de peso diário $(\mathrm{g})$ e da espessura de toucinho $(\mathrm{cm})$, para as raças Large White, Landrace e Duroc, são apresentadas na Tabela 2. Os gráficos das tendências dos valores genéticos são apresentados nas Figuras 1, 2 e 3.

As estimativas da tendência genética anual do peso aos 70 dias foram negativas, para as três raças. $\mathrm{O}$ progresso genético, estimado para peso aos 70 dias, foi de $-0,22 ;-0,24 ; \mathrm{e}-0,57 \mathrm{~kg} / \mathrm{ano}$, para as raças Large White, Landrace e Duroc, respectivamente, resultados que diferem dos de KUHLERS e JUNGST (1991), que encontraram tendência genética positiva para peso aos 70 dias.

O resultado obtido neste trabalho, para a raça Duroc, pode ser atribuído à baixa média de peso ajustado aos 70 dias $(19,0 \mathrm{~kg})$. Assim, possivelmente, os animais com menor peso ao final da creche apresentaram ganho de peso compensatório durante o teste (30 a $90 \mathrm{~kg}$ ). As correlações genéticas entre peso aos 70 dias e ganho de peso diário foi negativa $(-0,47)$ e entre peso aos 70 dias e espessura de toucinho foi positiva $(0,47)$. Assim, em razão de a ênfase na seleção ter sido maior no aumento no ganho de peso diário e na redução da espessura de toucinho, houve redução no peso aos 70 dias.

$\mathrm{Na}$ raça Landrace, apesar de a tendência também ser negativa, a redução no peso aos 70 dias foi menos intensa do que na raça Duroc, pois, para a raça Landrace, a correlação entre peso aos 70 dias e ganho de peso diário foi próxima a zero $(0,08)$. Com isso, houve menor pressão sobre o peso aos 70 dias.

Na raça Large White, essa tendência de redução no peso aos 70 dias é mais difícil de ser explicada, pois as correlações entre essa característica e ganho de peso diário e espessura de toucinho foram altas e positivas, 0,46 e 0,48 , respectivamente. A maior ênfase dada à redução na espessura de toucinho do que no aumento do ganho de peso diário, em razão da necessidade de se obter um animal com mais carne, talvez tenha resultado nessa redução no peso aos 70 dias.

As estimativas de tendências genéticas anuais para ganho de peso diário foram positivas para as três raças e estão de acordo com o objetivo de um programa de melhoramento genético de suínos, que é aumento no ganho de peso diário. O progresso genético, estimado pelo ganho de peso diário, foi de 14,11 ; 9,81; e 2,75 g/ano, para as raças Large White, Landrace e Duroc, respectivamente. Os valores encontrados para as raças Large White e Landrace foram maiores que os obtidos por FERRAZ e JOHNSON (1993), HOFER et al. (1992) e McKAY (1990), enquanto o valor obtido para a raça Duroc foi menor que os encontrados por esses autores.

As estimativas de tendências genéticas anuais para espessura de toucinho foram negativas para as três raças, o que também está de acordo com o objetivo de um programa de melhoramento genético para esta característica, que é sua redução. O progresso genético, estimado pela espessura de toucinho, foi de $-0,07 ;-0,05$; e $-0,04 \mathrm{~cm} / \mathrm{ano}$, para as raças Large White, Landrace e Duroc, respectivamente, valores estes maiores que os encontrados por FERRAZ e JOHNSON (1993), KAPLON et al. (1991) e HUDSON e KENNEDY (1985) e próximos aos alcançados por DAVID et al. (1985) e McKAY (1990).

Tabela 2 - Estimativas das tendências genéticas anuais $(\hat{b})$ e respectivos desvios-padrão $(s(\hat{b}))$ das características

Table 2 - Estimates of annual genetic trends $(\hat{\mathrm{b}})$ and respective standard deviations $(s(\hat{\mathrm{b}}))$ by traits

\begin{tabular}{|c|c|c|c|}
\hline \multirow[b]{2}{*}{$\begin{array}{l}\text { Característica } \\
\text { Trait }\end{array}$} & \multicolumn{3}{|c|}{$\begin{array}{l}\text { Raça } \\
\text { Breed }\end{array}$} \\
\hline & Large White & Landrace & Duroc \\
\hline $\begin{array}{l}\text { Peso aos } 70 \text { dias }(\mathrm{kg}) \\
\text { Weight at } 70 \text { d-old }(\mathrm{kg})\end{array}$ & $-0,22 \pm 0,12$ & $-0,24 \pm 0,09$ & $-0,57 \pm 0,11$ \\
\hline $\begin{array}{l}\text { Ganho de peso diário }(\mathrm{g}) \\
\text { Average daily gain }(\mathrm{g})\end{array}$ & $14,11 \pm 0,56$ & $9,81 \pm 3,45$ & $2,75 \pm 2,32$ \\
\hline $\begin{array}{l}\text { Espessura de toucinho }(\mathrm{cm}) \\
\text { Backfat thickness }(\mathrm{cm})\end{array}$ & $-0,07 \pm 0,01$ & $-0,05 \pm 0,003$ & $-0,04 \pm 0,01$ \\
\hline
\end{tabular}




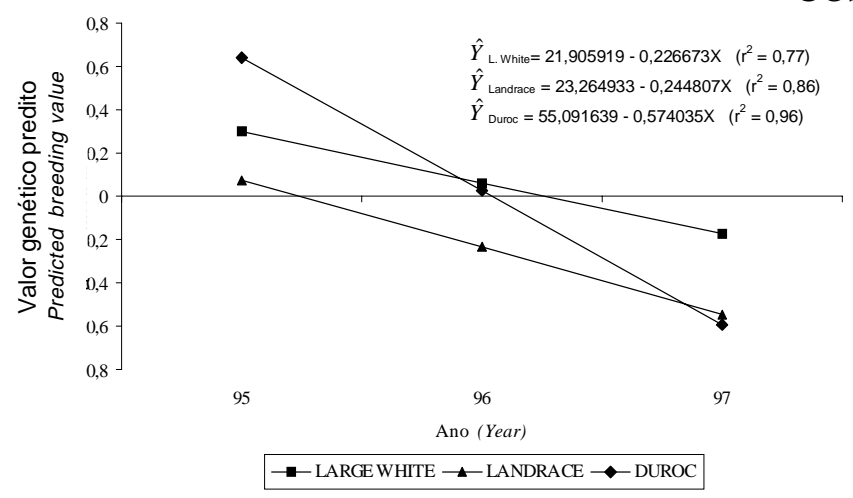

Figura 1 - Tendência genética do peso ajustado para 70 dias, calculada por meio da análise de regressão.

Figure 1 - Genetic trend of adjusted weight at $70 \mathrm{~d}$-old estimated by regression analysis.

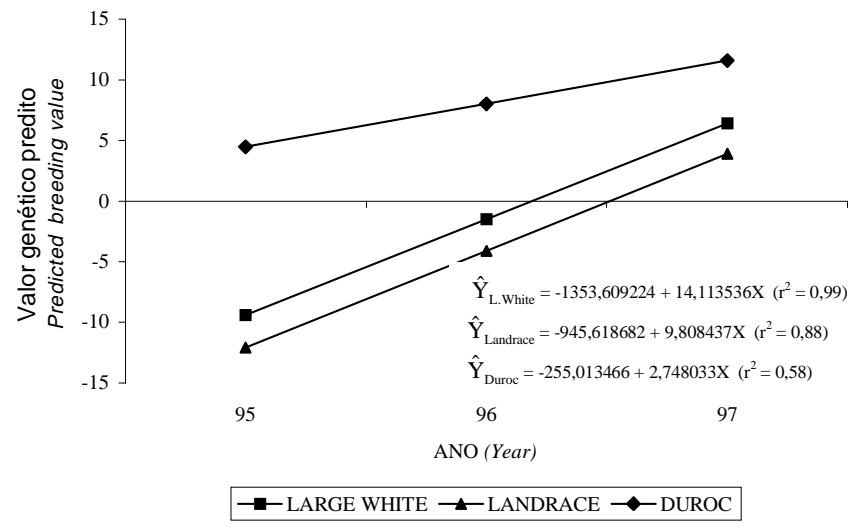

Figura 2 - Tendência genética do ganho de peso diário, calculada por meio da análise de regressão.

Figure 2 - Genetic trend of average daily gain estimated by regression analysis.

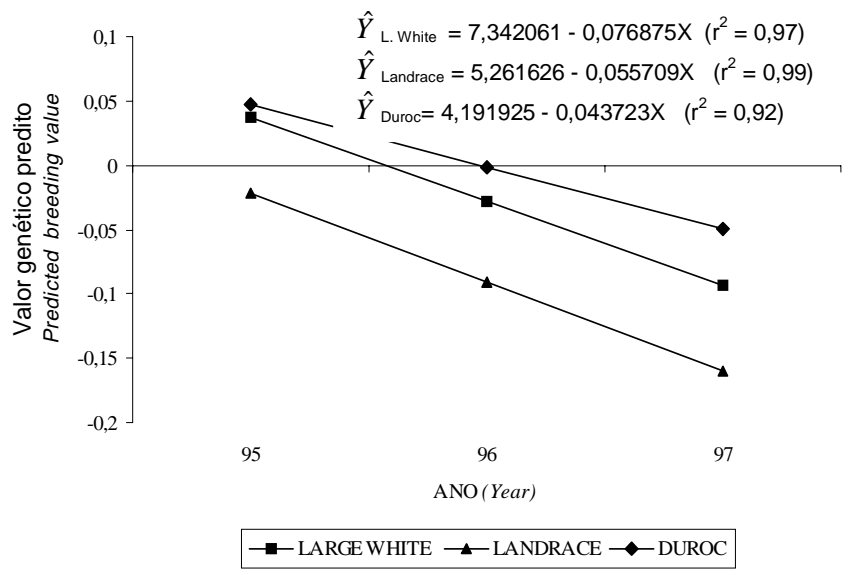

Figura 3 - Tendência genética da espessura de toucinho, calculada por meio da análise de regressão.

Figure 3 - Genetic trend of backfat thickness estimated by regression analysis.

\section{Conclusões}

As tendências genéticas positivas para ganho de peso diário e negativas para espessura de toucinho estão de acordo com os objetivos do programa de melhoramento, que são aumento no ganho de peso diário e redução na espessura de toucinho, assim como com as correlações genéticas entre essas características.

Dadas as tendências genéticas negativas e indesejáveis obtidas para peso aos 70 dias de idade, devem-se realizar mais trabalhos que utilizem o peso do animal na saída da creche, com vistas a verificar se a seleção para aumento desse peso não reduziria o progresso genético para ganho de peso diário e espessura de toucinho, para que se possa recomendar ou não a sua inclusão em programas de melhoramento, caso a préseleção, com base na taxa de crescimento, seja feita no período de saída do animal da creche. 


\section{Agradecimento}

À empresa COOPERCENTRAL, pela cessão dos dados, que possibilitaram a execução deste trabalho.

\section{Referências Bibliográficas}

BLAIR, H.T., POLLAK, E.J. 1984. Estimation of genetic trend in a selected population with and whithout the use of a control population. J. Anim. Sci., 58(4):878-86.

BOLDMAN, K.G., KRIESE, L.A., VAN VLECK, L.D. et al. 1995. A manual for use of MTDFREML. A set of programs to obtain estimates of variances and covariances [DRAFT]. Lincoln: USDA/ARS. 120p.

DAVID, P.J., JOHNSON, R.K., SOCHA, T.E. 1985. Selection practices and genetic and phenotypic trends estimated from Nebraska SPF swine field records. J. Anim. Sci., 61(6): 1411-20.

FERRAZ, J.B.S., JOHNSON, R.K. 1993. Animal model estimation of genetic parameters and response to selection for litter size and weight, growth and backfat in closed seedstock populations of Large White and Landrace swine. J. Anim. Sci., 71(4):850-58.

HILL, W.G. 1972a. Estimation of realized heritabilities from selection experiments. I. Divergent selection. Biometrics, 28(3):747-765.

HILL, W.G. 1972b. Estimation of realized heritabilities from selection experiments. II. Selection in one direction. Biometrics, 28(3):767-780.
HOFER, A., HAGGER, C., KUNZI, N. 1992. Genetic evaluation of on-farm tested pigs using na animal model. II. Prediction of breeding values with a multiple trait model. Livest. Prod. Sci., 30:83.

HUDSON, G.F.S., KENNEDY, B.W. 1985. Genetic evaluation of swine for growth rate and backfat thickness. J. Anim. Sci., 61(1):83-91.

KAPLON, M.J., ROTHSCHILD, M.F., BERGER, P.J. et al. 1991. Genetic and phenotypic trends in Polish Large White nucleus swine herds. J. Anim. Sci., 69(2):551-558.

KUHLERS, D.L., JUNGST, S.B. 1991. Mass selection for increased 200-day weight in a closed line of Duroc pigs. J. Anim. Sci., 69(2):507-516.

McKAY, R.M. 1990. Responses to index selection for reduced backfat thickness and increased growth rate in swine. Can. J. Anim. Sci., 70:973.

SORENSEN, D.A., KENNEDY, B.W. 1984. Estimation of response to selection using least-square and mixed model methodology. J. Anim. Sci., 58(5):1097-1106.

Recebido em: 30/06/99

Aceito em: 07/11/00 